\title{
An Update on HIV-1 Protease Inhibitor Resistance
}

John D Baxter ${ }^{1 *}$, William M Chasanov ${ }^{1}$ and Jessica L Adams ${ }^{2}$

${ }^{1}$ Cooper University Hospital/Cooper Medical School of Rowan University, NJ, USA

${ }^{2}$ Philadelphia College of Pharmacy, University of the Sciences, PA, USA

\begin{abstract}
Protease inhibitors (PIs) are a potent class of antiretroviral agents which have been used to successfully treat many patients with HIV disease. As with other classes of antiretroviral agents, drug resistance has been described with each of the PIs. PI resistance can occur in newly infected individuals due to transmission of drug resistant strains or, more commonly, evolves from the selective pressure of antiretroviral therapy. When present, PI resistance results in an increased likelihood of drug failure. Individual protease gene mutations have been identified which are associated with phenotypic resistance to these agents. However, protease mutations can exert different effects on individual PIs, as some will produce resistance to one agent and may enhance susceptibility to another. In patients failing PIs, particularly in the setting of prior treatment with multiple agents, protease resistance patterns may be complex and result in broad cross-resistance to the class. Strategies to limit the development of resistance to these agents and manage its occurrence are important for the on-going care of individuals with HIV infection.
\end{abstract}

Keywords: Human immunodeficiency virus (HIV); Protease inhibitor; HIV drug resistance

\section{Introduction}

Protease inhibitors (PIs) were initially introduced in the mid-1990s and when used in combination with nucleoside reverse transcriptase inhibitors (NRTI), ushered in the era of highly active antiretroviral therapy (HAART). These agents dramatically altered the treatment of HIV infection, enabling suppression of viral replication to undetectable levels and significantly impacting disease progression and mortality. However, HIV-1 drug resistance has been observed with all the PIs and the genetic basis of resistance has been well described [1]. The accumulation of multiple mutations is often required to produce PI resistance and there is variability of resistance patterns within the PI class. Some PIs require fewer mutations to confer resistance compared with others. In addition, the development of broad cross-resistance to multiple agents in this class presents a challenge in clinical practice.

PIs act by preventing the HIV protease enzyme from cleaving the gag protein, an essential step in the viral maturation process. PIs do this by binding to the active site or pocket of the protease enzyme rendering it unable to cleave the gag protein precursors into smaller viral proteins (Figure 1). PI resistance is usually associated with the development of

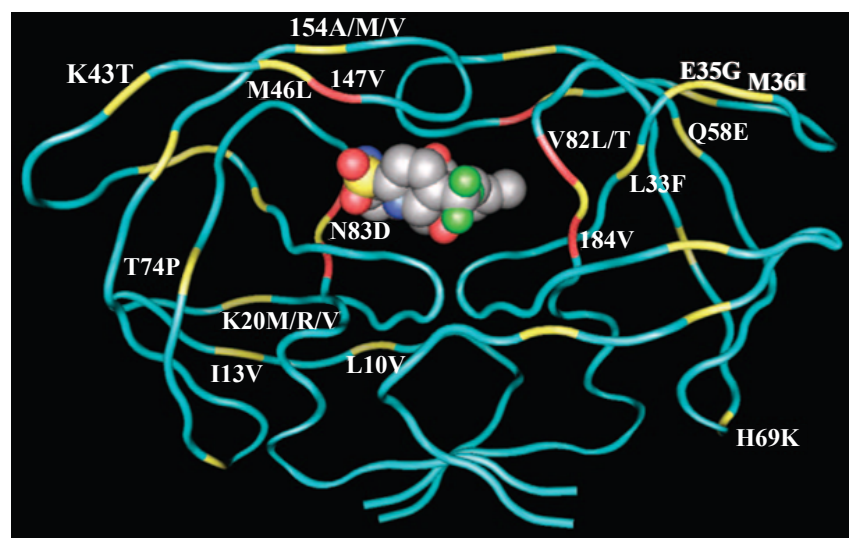

Figure 1: HIV-1 protease enzyme with the protease inhibitor tipranavir binding the active site. Amino acid changes associated with tipranavir resistance noted in the backbone of the protease enzyme [52] primary or secondary mutations in the protease gene and can also be due to mutations occurring outside of protease in the gag cleavage and non-cleavage sites. Primary mutations typically occur in the active site of the protease enzyme and may be sufficient to confer resistance to select PIs. Secondary mutations can enhance resistance to PIs or may be compensatory, restoring activity of the viral protease and increasing viral replicative capacity. The accumulation of multiple mutations alters the protease binding pocket and leads to increasing and broadened PI resistance. Specific resistance mutations have been identified with each of the individual PIs. Strategies to limit PI cross-resistance and to manage its occurrence include pharmacokinetic boosting to produce high PI drug concentrations resulting in a strong barrier against viral resistance, the use of second generation PIs including, atazanavir darunavir, fosamprenavir, lopinavir, and tipranavir, and exploiting the occurrence of HIV-1 hypersusceptibility to certain agents in this class.

Goals of antiretroviral therapy (ART) are to achieve sustained virologic suppression, reduce HIV/AIDS-related complications, and improve quality of life for those individuals living with HIV [2,3]. The initial widespread use of PIs as a component of HAART was associated with a marked reduction in AIDS related complications and death [4-6]. Guidelines for treatment of HIV issued by the US Department of Health and Human Services (DHHS) and the International AIDS Society (IAS)-USA Panel include PI-based antiretroviral regimens as recommended or alternative treatment options [2,3]. For treatment experienced patients failing therapy, these guidelines also recommend boosted PI containing regimens.

Compared to the other antiretroviral classes, PIs generally present a high genetic barrier against viral resistance and may remain active

*Corresponding author: John D Baxter, M.D., Professor of Medicine, Division of Infectious Diseases, Cooper University Hospital, Cooper Medical School of Rowan University, 401 Haddon Avenue, Room 270, Camden, NJ 08103-5609, Tel: (856) 757-7767; Fax: (856) 757-7803; E-mail: baxter@rowan.edu

Received May 05, 2016; Accepted May 19, 2016; Published May 25, 2016

Citation: Baxter JD, Chasanov WM, Adams JL (2016) An Update on HIV-1 Protease Inhibitor Resistance. J AIDS Clin Res 7: 581. doi:10.4172/2155-6113.1000581

Copyright: $\odot 2016$ Baxter JD, et al. This is an open-access article distributed under the terms of the Creative Commons Attribution License, which permits unrestricted use, distribution, and reproduction in any medium, provided the original author and source are credited. 
agents even in the presence of one or more protease mutations [7-12]. Single mutations alone are usually not sufficient to significantly reduce susceptibility for most PIs. Typically, the accumulation of multiple mutations is required to confer resistance to these agents. Most PIs are currently used with either ritonavir or cobicistat for pharmacologic boosting which can often overcome low-level PI resistance.

Non-adherence to PI based regimens has been associated with the risk of developing protease resistance [13]. Those individuals with a high level of adherence to PI based regimens are less likely to fail therapy with drug resistant HIV strains. In contrast, those patients who have difficulty with adherence to prior PI based regimens have an increased risk of resistance and virologic failure to other drugs in the PI class [14]. Of note, pharmacokinetic boosting of PIs allows patients with less than $100 \%$ adherence to have a significantly reduced risk of developing resistance compared to those receiving unboosted PI regimens $[15,16]$.

Many studies have described the prevalence of transmitted drug resistance in treatment-naive patients, which varies by geographic region [17-20]. It is currently recommended that resistance testing be performed before initiating ART in the setting of either acute or chronic infection [2,3]. If transmitted drug resistance is detected in a treatment-naïve individual this has implications for selection of the initial antiretroviral regimen, as three fully active agents should be prescribed for first-line therapy. Transmitted drug resistance may also increase the risk of virologic failure in subsequent regimens and impact future treatment options $[7,10,21,22]$. Fortunately, in most regions of the world the prevalence of transmitted drug resistance to PIs has been low compared to NRTIs and non-nucleoside reverse transcriptase inhibitors (NNRTIs) [23].

\section{The Genetics of PI Resistance}

To optimally use PI agents as an effective component of ART, it is important to understand the origins and mechanisms of PI resistance. Mutations conferring resistance to PIs may occur in different regions of the protease gene $[8,10,24-29]$. These regions include areas within the active site where the PI binds to the protease enzyme, as well as residues outside the active site. Mutations can also occur in the gag cleavage sites and non-cleavage sites which are areas entirely outside of the protease gene domain. Mutations occurring within the active binding site of protease result in conformational changes which disrupts PI binding to the enzyme $[8,24]$. These mutations in the protease binding site are termed primary or major protease mutations and have the greatest impact on PI susceptibility. Primary mutations, which typically occur in the protease enzyme pocket, disrupt hydrogen bonding between the PI molecule and the active site amino acid residues (Figure 1). These mutations prevent PI binding allowing the protease enzyme to continue to cleave the gag precursor polyproteins and thereby complete the viral lifecycle.
In contrast, mutations involving amino acid residues occurring outside of the active binding site are referred to as secondary or minor mutations $[8,24]$. Secondary mutations alone do not usually cause decreased susceptibility to PIs. Instead, secondary mutations typically occur following the development of primary protease mutations and enhance the degree of PI resistance. Occasionally, the accumulation of multiple secondary mutations may result in reduced susceptibility to some PIs. Secondary mutations are usually compensatory, restoring protease enzyme activity or reversing viral fitness defects produced by primary mutations [8]. Secondary mutations often involve amino acid substitutions at positions associated with naturally occurring polymorphisms, such as 36 and 71 of protease $[8,24]$.

Mutations occurring outside of the protease gene can also contribute to the development of PI resistance. Viral evolution of mutations and insertions at the gag cleavage sites and non-cleavage sites occurring after exposure to PIs has been well described. These mutations are associated with the restoration of protease enzyme activity and viral replicative capacity that is typically compromised in multi-PI-resistant variants [8,10,30-34]. Gag cleavage site mutations typically occur along with primary and secondary protease mutations, but have been reported to occasionally be the sole mechanism responsible for resistance to PIs in the absence of any protease mutations [35]. Mutations in gag cleavage and non-cleavage sites lie completely outside of the protease gene region, representing changes in the polyprotein substrate upon which the protease enzyme acts. It is noteworthy that PIs are the only antiretroviral drug class for which resistance-associated amino acid substitutions have been identified which are external to the targeted enzyme or protein [36]. Although the gag cleavage and non-cleavage site mutations represent another mechanism of PI resistance and as they occur outside of the protease region, they are not generally detected on standard HIV genotypic resistance testing.

\section{Resistance to specific PIs}

The understanding of HIV drug resistance has evolved over time and continues to change as new findings emerge. It is important for HIV providers to stay current with the latest findings on primary and secondary protease mutations associated with specific agents to optimize regimen selection in individual patients. Specific protease mutational patterns associated with resistance to individual PIs are listed in Table 1.

The L90M mutation is observed during treatment failure involving a number of PIs, but historically has been commonly selected by exposure to saquinavir. Reduced susceptibility to saquinavir is less frequently associated with the G48V mutation, which confers high-level (10fold) resistance to this agent. There are multiple secondary mutations associated with saquinavir, including L10I/R/V, L24I, I54V/L, A71V/T,

\begin{tabular}{|c|c|c|}
\hline Drug & Primary resistance mutations & Secondary resistance mutations \\
\hline Atazanavir +/- ritonavir & I50L, I84V, N88S & $\begin{array}{l}\text { L10I/F/V/C, G16E, K20R/M/I/T/V, L24I, V32I, L33I/F/V, E34Q, M36I/L/V, M46I/L, G48V, F53L/Y, I54L/V/M/ } \\
\text { T/A, D60E, I62V, I64L/M/V, A71V/I/T/L, G73C/S/T/A, V82A/T/F/I, I85V, L90M, I93L/M }\end{array}$ \\
\hline Darunavir/ritonavir & I47V, I50V, I54M/L, L76V, I84V & V11I, V32I, L33F, T74P, L89V \\
\hline Fosamprenavir/ritonavir & $150 \mathrm{~V}, 184 \mathrm{~V}$ & L10F/I/R/V, V321, M46I/L, I47V, I54L/V/M, G73S, L76V, V82A/F/S/T, L90M \\
\hline Indinavir/ritonavir & M46I/L, V82A/F/T, I84V & L10I/R/V, K20M/R, L24I, V32I, M36I, I54V, A71V/T, G73S/A, L76V, V77I, L90M \\
\hline Lopinavir/ritonavir & V32I, I47V/A, L76V, V82A/F/T/S & L10F/I/R/V, K20M/R, L24I, L33F, M46I/L, I50V, F53L, I54V/L/A/M/T/S, L63P, A71V/T, G73S, I84V, L90M \\
\hline Nelfinavir & D30N, L90M & L10F/I, M36I, M46I/L, A71V/T, V77I, V82A/F/T/S, I84V, N88D/S \\
\hline Ritonavir & V82A/F/T/S, 184V & L10F/I/R/V, K20M/R, V32I, L33F, M36I, M46I/L, I50V, I54V/L, A71V/T, V77I, L90M \\
\hline Saquinavir/ritonavir & G48V, L90M & L10I/R/V, L24I, I54V/L, I62V, A71V/T, G73S, V77I, V82A/F/T/S, I84V \\
\hline Tipranavir/ritonavir & 147V, Q58E, T74P V82L/T, N83D, 84V & L10V, L33F, M36I/L/N, K43T, M46L, I54A/M/V, H69K/R, L89I/M/V \\
\hline
\end{tabular}

Table 1: Mutations in the protease gene associated with resistance to protease inhibitors $[24,70,71]$. 
I62V, G73S, V77I, V82A/F/T/S and I84V. The V82A mutation has also been reported in patients failing saquinavir-containing regimens following the emergence of the G48V mutation [8]. In contrast to other PIs, saquinavir exposure has not been associated with mutations at position 46 of protease, a common site for primary and secondary mutations with these agents $[8,12,28,37-39]$.

The V82A/F/T mutation is frequently selected in individuals failing therapy on indinavir and ritonavir. This mutation alone may be sufficient to result in phenotypic resistance to both agents. When present with other primary and secondary PI resistance mutations, the V82A/F/T mutation contributes to resistance to many of the other PIs, including lopinavir/ritonavir, atazanavir, fosamprenavir, nelfinavir, and saquinavir [8]. Primary mutations associated with indinavir resistance include M46I/L, V82A/F/T, and I84V [24]. Ritonavir was used as a single PI after initial FDA approval and resistance patterns with this agent are similar to those associated with indinavir resistance. Ritonavir is currently used as a pharmacologic booster of other PIs which has greatly reduced the emergence of mutations selecting for resistance to those PIs as well as to ritonavir $[15,16]$. In fact, with boosted PI regimens it is unusual to detect PI resistance mutations in patients experiencing early first regimen failure.

Patients failing on nelfinavir-based regimens generally demonstrate one of two resistance pathways $[24,40]$. The most common is the D30N mutation, which is the primary mutation associated with nelfinavir failure and is not associated with resistance to the other PIs. Less commonly nelfinavir resistance occurs via the L90M pathway which may be associated with cross-resistance to other PIs. In contrast, the D30N mutation is associated with impaired viral fitness, and may confer enhanced susceptibility to other PIs [40].

The primary protease mutations associated with resistance to fosamprenavir are I50V (most common) and I84V [24]. In the NEAT trial, continued treatment with unboosted fosamprenavir in a patient with virologic failure was associated with the development of the I50V mutation $[41,42]$. Little to no cross-resistance to other PIs was observed in association with fosamprenavir resistance in this study. In the SOLO trial, ritonavir-boosted fosamprenavir was not associated with the emergence of fosamprenavir resistance mutations [15,41]. Among patients with virologic failure in this study, reduced susceptibility to fosamprenavir was not seen. However, fosamprenavir resistance may occur with prolonged drug exposure in the presence of virologic failure. The mutations most closely associated with fosamprenavir resistance are listed in Table 1.

Reduced susceptibility to lopinavir/ritonavir is typically associated with multiple primary and secondary protease mutations (Table 1) [16]. The presence of six of these mutations is associated with reduced virologic response to lopinavir/ritonavir, and accumulation of seven or eight mutations usually results in full resistance to this combination [24]. The inhibitory quotient, which integrates drug exposure and genotypic resistance, has been found to be a better predictor of response to lopinavir/ritonavir in highly treatment-experienced patients compared to genotypic or pharmacologic measures alone [43]. Substitutions at positions 46, 54 and 82 of protease are important in the development of high-level resistance to lopinavir and have been frequently observed in patients failing on this agent [37]. The I50V, which is considered a secondary mutation, can also contribute to lopinavir/ritonavir resistance [41,44]. Although resistance to lopinavir has been associated with development of multiple protease mutations, there is evidence that specific mutations, particularly I47A, can result in high level resistance $[24,45,46]$. The I47A mutation usually occurs with the V32I mutation and produces high level resistance to lopinavir/ritonavir. In addition, the L76V in combination with three other protease mutations substantially reduces susceptibility to lopinavir/ritonavir [24].

Resistance to atazanavir is associated with the I50L (most commonly), I84V, and N88S primary mutations [24]. In patients receiving unboosted atazanavir in an initial antiretroviral regimen, the I50L mutation has been identified as the signature mutation with this agent $[47,48]$. Pooled data from three trials with unboosted atazanavir-containing regimens as initial therapy in treatment naïve patients revealed that in those failing therapy, $15-48 \%$ had the I50L mutation $[47,48]$. Interestingly, isolates containing the I50L mutation had atazanavir-specific resistance but they also demonstrated enhanced susceptibility to other PIs $[38,49]$. A case report of an isolate with the N88S mutation noted high-level resistance to atazanavir with low replicative capacity in an antiretroviral-naïve patient who had no other primary protease mutations present [50]. An analysis from the Monogram database of isolates that contained only the N88S mutation found cross-resistance to atazanavir, nelfinavir and indinavir and hypersusceptibility to amprenavir. In extensively PI experienced patients receiving an atazanavir-containing regimen who develop atazanavir resistance, the $\mathrm{I} 84 \mathrm{~V}, \mathrm{~L} 90 \mathrm{M}, \mathrm{A} 71 \mathrm{~V} / \mathrm{T}, \mathrm{N} 88 \mathrm{~S} / \mathrm{D}$ and M46I protease mutations are most typically seen $[47,49]$. Of note, higher atazanavir levels associated with pharmacokinetic boosting increases the number of PI mutations required for loss of activity [24].

Tipranavir and darunavir usually have activity to HIV-1 exhibiting resistance to the other PIs. For both agents, numerous mutations are usually necessary to confer resistance. The understanding of tipranavir resistance has evolved over time. In vitro, tipranavir was found to suppress viral replication of laboratory variants and clinical isolates with high-level resistance to multiple PIs [51]. Initial work analyzing data from treatment-experienced patients showed that multiple tipranavir associated mutations were required for reduced response to tipranavir/ ritonavir. These included L10V, I13V, K20M/R/V, E33G, M36I, K43T, M46L, I47V, I54A/M/V, Q58E, H69K, T74P and N83D, and I84V; also referred to as the tipranavir score mutations [52]. Regression analysis from phase III trials of tipranavir/ritonavir in treatment-experienced patients revealed that each point (representing one of these mutations) in the tipranavir score was associated with a $0.16 \mathrm{log}$ copies/ml lower virologic response to tipranavir at week 24 of treatment $[52,53]$. Subsequent validated analyses of clinical trials data allowed for a refined "weighted" mutation score to be developed [54]. Assigned weights of the new score were T74P, V82L/T, N83D and I47V (+4), Q58E and I84V (+3), M36I, K43T and I54A/M/V (+2), L10V, E33F and M46L (+1), L24I and L76V (-2), I50L/V (-4), and I54L (-6). Positive weighted mutations are associated with reduced susceptibility and negative score mutations are associated with enhanced activity with tipranavir/ ritonavir [54,55]. Sum scores of $\leq 3$ are defined as susceptible, scores $>3$ and $\leq 10$ partially susceptible, and $\geq 11$ resistant.

Darunavir, which was initially approved for use in treatmentexperienced patients, has activity against both laboratory and clinical isolates with high-level resistance to multiple PIs including nelfinavir, indinavir, ritonavir, amprenavir, saquinavir and lopinavir [56]. Analyses of pooled baseline and on-treatment resistance data from Phase III clinical trials has enhanced our understanding of boosted darunavir activity in patients with prior PI exposure [57]. In these studies, patients with a history of PI, NNRTI and NRTI experience, at least one primary PI mutation, and documented virologic failure were randomized to either boosted darunavir or an investigator-selected control protease inhibitor, along with an optimized background 
regimen. Reduced response to darunavir was associated with eleven mutations: V11I, V32I, L33F, I47V, I50V, I54L, I54M, T74P, L76V, I84V, and L89V. The presence of two or more of these mutations was associated with a decreased virologic response to darunavir/ritonavir. Subsequent analyses showed that in patients not taking enfuvirtide de novo, the proportion achieving an HIV-1 RNA viral load $<50$ copies/ $\mathrm{ml}$ at 96 weeks was $59 \%, 29 \%$, and $12 \%$ when the baseline genotype had $0-1,2$, and $\geq 3$ of these darunavir associated mutations. As shown in Table 1, six of the darunavir associated mutations are considered primary mutations and are associated with the greatest reduction in susceptibility. Of note, one of these primary mutations affecting response to darunavir/ritonavir is $\mathrm{I} 50 \mathrm{~V}$, which is also associated with fosamprenavir resistance. Furthermore, the negative impact of mutations I47V, I54M, T74P, and I84V and a positive impact of V82A on virologic response to darunavir/ritonavir has been demonstrated in two independent data sets $[58,59]$. A recent retrospective study was conducted of 880 treatment experienced adult patients initiating a darunavir containing antiretroviral salvage regimen after having previously failed PIs. Patients were selected and data was collected from large European databases of HIV infected patients. The association of mutations with week 8 HIV RNA change from baseline was analyzed. The mutations L10F, V11L, I54M, T74P, and V82I were found to negatively impact viral load change, while the mutations K20T, E34D, I64L, V82A, I85V, and I93L had a positive impact on viral load response. The authors point out that established darunavir associated mutations (such as the primary mutations I47V, I50V, L76V, and I84V) were likely not found to be associated with a decreased response in this particular study because patients with evidence of those mutations would likely not have been started on a darunavir containing salvage regimen [60].

\section{Cross-resistance}

Cross-resistance between PIs has been a challenging problem in clinical practice $[9,10,61]$. Even though boosted PIs generally maintain a high genetic barrier against viral resistance, the evolution of protease resistance over time may still occur with continued drug exposure, and can result in reduced response to other PI-based regimens.

The accumulation of protease resistance mutations does not occur in a random fashion. Rather, these mutations accrue based on pathways associated with specific drug exposure $[9,10,61]$. Experiments involving in vitro serial passage of laboratory HIV-1 strains in the presence of individual PIs demonstrate that protease resistance mutations accumulate in a sequential pattern unique to each particular drug. As amino acid substitutions in protease accumulate, the likelihood that these mutations will affect response to multiple agents within the PI class also increases. This phenomenon produces overlapping genotypic resistance patterns seen with different PIs and ultimately results in broad phenotypic cross-resistance.

The original genetic background of the infecting virus and the preexistence of polymorphisms in antiretroviral-naïve patients may also be important determinants of resistance to individual PIs as well as cross-resistance to other agents in this class $[9,10]$. Polymorphisms described in some treatment-naive patients may lower the genetic barrier to PI resistance. For example, the M36I substitution when present prior to the initiation of ART, may predispose patients to the development of the L90M mutation once a PI containing regimen is begun.

Patients who experience virologic failure on a second PI may have mutational imprinting, in which the evolution of viral resistance mutations reflects exposure to the initial PI [60]. A study of 153 patients failing ART found that those patients who were on their second PIbased regimen often had signature mutations that were associated with their first PI-based regimen. This is likely due to the already mutated virus evolving additional resistance in a background of mutations reflective of the PI initially prescribed.

The degree to which PI cross-resistance is present in a population of HIV-infected individuals was shown in a study of matched genotypic and phenotypic resistance testing of $>6,000$ clinical HIV isolates $[9,10,62]$. Genotypic and phenotypic analysis demonstrated that $59 \%$ to $80 \%$ of the isolates that had decreased susceptibility to one PI exhibited cross-resistance to at least one other PI.

Because of the significant challenge posed by PI cross-resistance and its impact on therapeutic options, treatment strategies should reduce the opportunity for development of broad cross-resistance and manage it effectively when it occurs. Pharmacokinetic boosting to maintain a strong pharmacologic barrier against viral resistance and the use of PIs active against resistant strains can limit the likelihood of cross-resistance [10-12].

In HIV isolates that exhibit multiple protease mutations, prediction of cross resistance may be difficult using genotypic testing alone. Currently, for isolates with complex drug-resistance mutation patterns, particularly with PI resistance, it is recommended that phenotypic testing also be performed [3]. Phenotypic susceptibility testing can help define the extent of PI cross-resistance, determine which PIs remain active, and detect HIV hypersusceptibility to specific agents. A standard phenotype assay is a direct measure of drug susceptibility and requires growth of recombinant virus in cell culture. In contrast, the vircoTYPE or "virtual phenotype" was based on a genotype assay with interpretation of drug susceptibility that relied on a large matched genotype-phenotype database. The VircoTYPE assay is no longer commercially available as it was discontinued by Janssen Diagnostics in December, 2013.

\section{Hypersusceptibility}

Some protease mutations have been associated with increased viral susceptibility to other PIs rather than resulting in cross-resistance $[9,10,12]$. Hypersusceptibility is detected with phenotypic resistance assays and occurs when an HIV isolate exhibits a reduced fold change (FC=IC50 of a clinical isolate/IC50 of a susceptible control laboratory strain) compared to the biologic average FC for susceptible "wild-type" viruses. Wild-type susceptible HIV strains would typically have a FC of 1 for a particular protease inhibitor, while an isolate having a FC $<0.5$ would generally be considered hypersusceptible. Although there is limited clinical data, it appears that hypersusceptibility is associated with enhanced drug activity and can be considered in the selection of protease inhibitors for "salvage" or "rescue" regimens in treatment experienced patients failing therapy.

In vitro studies have demonstrated that the $\mathrm{N} 88 \mathrm{~S}$ protease mutation is associated with hypersusceptibility to amprenavir [63]. Clinical samples from 20 patients failing virologically on a regimen containing nelfinavir or indinavir showed increased susceptibility to amprenavir; this finding was strongly associated with the N88S mutation. Another study using amprenavir/ritonavir in patients failing prior PI-based therapy showed that amprenavir hypersusceptibility (defined as a $\mathrm{FC}<0.66)$ was associated with increased odds of achieving virologic suppression [64].

Hypersusceptibility to tipranavir has been demonstrated in clinical samples that are highly resistant to other PIs [54,55]. As described 
above, the mutations L24I, L76V, I50L/V, and I54L are associated with enhanced susceptibility and improved activity with tipranavir/ ritonavir. An analysis from the Phase III RESIST trials found that hypersusceptibility to tipranavir $(\mathrm{FC}<0.5)$ was associated with a more durable virologic response to tipranavir/ritonavir [55]. Of interest, some of these hypersusceptibility mutations for tipranavir represent primary resistance mutations for other protease inhibitors (ex. I50V, I54L, and L76V for darunavir). The L76V mutation has been associated with resistance to lopinavir, fosamprenavir, indinavir, and darunavir but appears to enhance susceptibility to tipranavir, atazanavir, and saquinavir due to differences in the hydrophobic binding of these agents in the S2 pocket of the protease enzyme.

The I50L mutation, which is uniquely associated with atazanavir resistance, has been demonstrated to produce hypersusceptibility to amprenavir, indinavir, lopinavir, nelfinavir, ritonavir and saquinavir $[12,48]$. Furthermore, isolates that were originally resistant to multiple PIs had increased susceptibility to these agents following treatment with atazanavir and emergence of the I50L mutation [65].

A study evaluating patients who initiated a darunavir containing antiretroviral regimen after having previously failed PIs found the mutations K20T, E34D, I64L, V82A, I85V and I93L to be correlated with improved rates of virologic response at 8 weeks [60]. The I64L, $\mathrm{I} 85 \mathrm{~V}$, and $193 \mathrm{~L}$ mutations are secondary mutations affecting atazanavir. The V82A mutation is a primary mutation for indinavir/ritonavir and lopinavir/ritonavir, and a secondary mutation for a number of PIs including atazanavir, fosamprenavir, nelfinavir, and saquinavir [24]. It has previously been shown to increase susceptibility to darunavir $[58,59]$.

When choosing subsequent PI-based regimens in treatmentexperienced patients, clinicians should consider the opportunities hypersusceptibility may offer and its implications for regimen selection. Although the predicted effects of mutations associated with hypersusceptibility to a particular PI can be incorporated into genotypic interpretative algorithms, it is best to confirm the presence of hypersusceptibility with a phenotypic resistance assay. Furthermore, in salvage therapy it is preferable to use three fully active agents in the new regimen to achieve virologic suppression, decrease evolution of resistant virus, and preserve future treatment options [3].

\section{Multi-PI Resistance}

As primary and secondary mutations associated with resistance to agents within the PI class accumulate, susceptibility to the majority of available PIs will often decrease [24]. Certain mutations are associated with broad resistance to the PI class. For example, the I84V mutation is associated with resistance to all the currently available protease inhibitors, although it is considered a primary mutation for some agents but not others (Table 1) [24]. One analysis found that the most frequent mutations identified in multi-PI resistant isolates were at positions 10, 54, 71, 77, 82, 84, and 90 [62]. A study of HIV isolates in patients failing PI-based regimens found that broad PI resistance was more commonly observed in those with exposure to multiple prior PIs [66]. Of those isolates with resistance to all first generation PIs, 53\% remained susceptible to either darunavir or tipranavir, while overall $5 \%$ of isolates exhibited broad resistance to all PIs. Multi-PI resistant viruses may also have mutations in the gag protease cleavage sites (the protease substrates), which may further enhance phenotypic resistance and increase viral fitness. In viruses that exhibit broad PI resistance with complex mutational patterns detected by genotypic testing, it is optimal to also obtain a phenotype to determine the extent of multi-PI resistance and to potentially identify residual PI drug activity.

\section{Selection of Protease Inhibitor Therapy}

The selection of the initial antiretroviral regimen provides the greatest chance for long-term success in optimally suppressing viral replication [67]. The most important factors in selecting the initial PI-based regimen include high potency and durability, favorable pharmacokinetics, good tolerability, a beneficial safety profile, and low potential for cross-resistance [12]. According to the DHHS guidelines panel, the preferred PI for initial therapy in adults and adolescents is ritonavir-boosted darunavir [3]. Favorable tolerability, safety and convenience with the initial regimen may also help promote a high level of adherence with treatment. Fortunately, initial therapy with boosted PIs is usually not associated with protease resistance at the time of first virologic failure given the potency and relatively high genetic barrier to resistance with these agents.

Selection of PIs in treatment-experienced patients should be based on genotypic and possibly phenotypic analyses of clinical isolates after virologic failure has been confirmed [18]. Resistance testing is a useful tool for identifying the agent in a current regimen to which the virus has developed resistance or reduced susceptibility, which may allow for the replacement of only that drug rather than the switching of the entire regimen. It is important when using a new PI in salvage or rescue therapy, that it is used with two other fully active agents identified by resistance testing. Typically, in treatment-experienced patients with PI resistance, the most active drugs are darunavir and tipranavir.

Another consideration in interpreting results from resistance testing is the possibility of viral reversion to wild type $[9,10,68]$. This occurs when ART is discontinued and selective drug pressure is removed. As a consequence, the major HIV-1 population in a particular patient shifts from predominantly drug resistant variants to wild type susceptible virus. Reversion to wild type is most commonly due to outgrowth of more replication-competent virus from the pool of latently infected $\mathrm{T}$ cells or it may occasionally result from a new amino acid substitution in the predominant replicating virus to produce a variant with improved fitness $[9,10]$. For this reason, it is optimal to perform resistance testing while the patient is on a failing regimen, as this will maximize the detection of resistance mutations and more accurately assess the extent of resistance to the current drug regimen.

In some individuals with multi-drug resistant (MDR) HIV, exhibiting broad resistance to multiple classes, effective options may be exhausted for these patients and their viral loads remain detectable on failing regimens. Treatment interruptions in patients with MDR virus are associated with elevations in viral load, rapid decline in CD4 T-cell counts, and disease progression $[68,69]$. In the setting of highly drug resistant HIV it is best to continue partially suppressive therapy, potentially including a PI to maintain drug resistance mutations associated with reduced viral fitness, as the only viable strategy to preserve immune function until new active agents become available.

\section{Conclusion}

PIs represent a potent class of antiretrovirals which have contributed to the success of HAART. Resistance has been well characterized for all available PIs and is associated with the accumulation of both primary and secondary mutations (Table 1). Given the high genetic barrier to resistance for most of these agents, multiple PI mutations are usually required to produce phenotypic drug resistance. Signature mutations associated with a particular PI may result in cross-resistance with other agents in the class or may be associated with hypersusceptibility. Patients with exposure to multiple PIs are more likely to have broadly 
PI resistant viruses, however, second generation PIs may retain activity against these strains.

For first regimen failure, genotypic testing is usually adequate to detect the presence of drug resistance. For subsequent regimen failure with viral isolates exhibiting complex PI drug-resistance mutation patterns, it is recommended that phenotypic resistance testing also be performed to better define the extent of protease inhibitor crossresistance, determine residual drug activity, and potentially detect hypersusceptibility. Strategies to prevent PI cross-resistance and to manage its occurrence involve pharmacokinetic boosting with either ritonavir or cobicistat to maintain a strong barrier against viral resistance, application of knowledge derived from drug resistance testing, and use of potent active agents in combination to maintain durable virologic suppression.

\section{References}

1. Wensing AM, van Maarseveen NM, Nijhuis M (2010) Fifteen years of HIV Protease Inhibitors: raising the barrier to resistance. Antiviral Res 85: 59-74.

2. Thompson MA, Aberg JA, Hoy JF, Telenti A, Benson C, et al. (2012) Antiretroviral treatment of adult HIV infection: 2012 recommendations of the International Antiviral Society-USA panel. JAMA 308: 387-402.

3. Panel on Antiretroviral Guidelines for Adults and Adolescents (2016) Guidelines for the use of antiretroviral agents in HIV-1-infected adults and adolescents. Department of Health and Human Services (DHHS).

4. Palella FJ Jr, Delaney KM, Moorman AC, Loveless MO, Fuhrer J, et al. (1998) Declining morbidity and mortality among patients with advanced human immunodeficiency virus infection. HIV Outpatient Study Investigators. N Engl J Med 338: 853-860.

5. Murphy EL, Collier AC, Kalish LA, Assmann SF, Para MF, et al. (2001) Highly active antiretroviral therapy decreases mortality and morbidity in patients with advanced HIV disease. Ann Intern Med 135: 17-26.

6. Jacobson MA, French M (1998) Altered natural history of AIDS-related opportunistic infections in the era of potent combination antiretroviral therapy. AIDS 12: S157-S163.

7. Clavel F, Hance AJ (2004) HIV drug resistance. N Engl J Med 350: 1023-1035

8. de Mendoza C, Soriano V (2004) Resistance to HIV protease inhibitors: mechanisms and clinical consequences. Curr Drug Metab 5: 321-328.

9. Kozal M (2004) Cross-resistance patterns among HIV protease inhibitors. AIDS Patient Care STDS 18: 199-208.

10. Kim R, Baxter JD (2008) Protease inhibitor resistance update: where are we now? AIDS Patient Care STDS 22: 267-277.

11. Schnell T, Schmidt B, Moschik G, Thein C, Paatz C, et al. (2003) Distinct crossresistance profiles of the new protease inhibitors amprenavir, lopinavir, and atazanavir in a panel of clinical samples. AIDS 17: 1258-1261.

12. Turner D, Schapiro JM, Brenner BG, Wainberg MA (2004) The influence of protease inhibitor resistance profiles on selection of HIV therapy in treatmentnaive patients. Antivir Ther 9: 301-314.

13. Baxter JD (2006) The experts speak. New issues in the challenge of HIV drug resistance. Interview by Vicki Glaser. AIDS Patient Care STDS 20: 737-740.

14. Gilliam BL, Chan-Tack KM, Qaqish RB, Rode RA, Fantry LE, et al. (2006) Successful treatment with atazanavir and lopinavir/ritonavir combination therapy in protease inhibitor-susceptible and protease inhibitor-resistant HIVinfected patients. AIDS Patient Care STDS 20: 745-759.

15. Gathe JC, Jr., Ive P, Wood R, Schurmann D, Bellos NC, et al. (2004) SOLO: 48-week efficacy and safety comparison of once-daily fosamprenavir/ritonavir versus twice-daily nelfinavir in naive HIV-1-infected patients. AIDS 18:15291537.

16. Kempf DJ, King MS, Bernstein B, Cernohous P, Bauer E, et al. (2004) Incidence of resistance in a double-blind study comparing lopinavir/ritonavir plus stavudine and lamivudine to nelfinavir plus stavudine and lamivudine. $J$ Infect Dis 189: 51-60.

17. Daar ES, Richman DD (2005) Confronting the emergence of drug-resistant HIV type 1: impact of antiretroviral therapy on individual and population resistance. AIDS Res Hum Retroviruses 21: 343-357.

18. Hirsch MS, Brun-Vézinet F, D'Aquila RT, Hammer SM, Johnson VA, et al. (2000) Antiretroviral drug resistance testing in adult HIV-1 infection: recommendations of an International AIDS Society-USA Panel. JAMA 283: 2417-2426.

19. Little SJ, Holte S, Routy JP, Daar ES, Markowitz M, et al. (2002) Antiretroviraldrug resistance among patients recently infected with HIV. N Engl J Med 347: 385-394.

20. Pillay D (2004) Current patterns in the epidemiology of primary HIV drug resistance in North America and Europe. Antivir Ther 9: 695-702.

21. Deeks SG (2003) Treatment of antiretroviral-drug-resistant HIV-1 infection. Lancet 362: 2002-2011.

22. Phillips AN, Dunn D, Sabin C, Pozniak A, Matthias R, et al. (2005) Long term probability of detection of HIV-1 drug resistance after starting antiretroviral therapy in routine clinical practice. AIDS 19: 487-494.

23. WHO (2015) HIV drug resistance surveillance guidance 2015 update. World Health Organization.

24. Wensing AM, Calvez V, Günthard HF, Roger P, Deenan P, et al. (2015) Update of the Drug Resistance Mutations in HIV-1: March 2013. Top Antivir Med. 23.

25. Rhee SY, Liu T, Ravela J, Gonzales MJ, Shafer RW (2004) Distribution of human immunodeficiency virus type 1 protease and reverse transcriptase mutation patterns in 4,183 persons undergoing genotypic resistance testing. Antimicrob Agents Chemother 48: 3122-3126.

26. Rhee SY, Fessel WJ, Zolopa AR, Hurley L, Liu T, et al. (2005) HIV-1 Protease and Reverse-Transcriptase Mutations: Correlations with Antiretroviral Therapy in Subtype B Isolates and Implications for Drug-Resistance Surveillance. Infect Dis 192: 456-465.

27. Svicher V, Ceccherini-Silberstein F, Erba F, Santoro M, Gori C, et al. (2005) Novel human immunodeficiency virus type 1 protease mutations potentially involved in resistance to protease inhibitors. Antimicrob Agents Chemother 49: 2015-2025.

28. Watkins T, Resch W, Irlbeck D, Swanstrom R (2003) Selection of high-leve resistance to human immunodeficiency virus type 1 protease inhibitors. Antimicrob Agents Chemother 47: 759-769.

29. Winters MA, Merigan TC (2005) Insertions in the human immunodeficiency virus type 1 protease and reverse transcriptase genes: clinical impact and molecular mechanisms. Antimicrob Agents Chemother 49: 2575-2582.

30. Boden D, Markowitz M (1998) Resistance to human immunodeficiency virus type 1 protease inhibitors. Antimicrob Agents Chemother 42: 2775-2783.

31. Gatanaga H, Suzuki Y, Tsang H, Yoshimura K, Kavlick MF, et al. (2002) Amino acid substitutions in $\mathrm{Gag}$ protein at non-cleavage sites are indispensable for the development of a high multitude of HIV-1 resistance against protease inhibitors J Biol Chem 277: 5952-5961.

32. Mammano F, Trouplin V, Zennou V, Clavel F (2000) Retracing the evolutionary pathways of human immunodeficiency virus type 1 resistance to protease inhibitors: virus fitness in the absence and in the presence of drug. $J$ Virol 74 8524-8531.

33. Myint L, Matsuda M, Matsuda Z, Yokomaku Y, Chiba T, et al. (2004) Gag noncleavage site mutations contribute to full recovery of viral fitness in protease inhibitor-resistant human immunodeficiency virus type 1. Antimicrob Agents Chemother 48: 444-452.

34. Tamiya S, Mardy S, Kavlick MF, Yoshimura K, Mistuya H (2004) Amino acid insertions near Gag cleavage sites restore the otherwise compromised replication of human immunodeficiency virus type 1 variants resistant to protease inhibitors. J Virol 78: 12030-12040.

35. Nijhuis M, van Maarseveen NM, Schipper P (2004) Novel HIV drug resistance mechanism leading to protease inhibitor $(\mathrm{PI})$ resistance in response to a high genetic barrier PI in vitro. Antivir Ther. 9: S42.

36. Miller V (2001) International perspectives on antiretroviral resistance. Resistance to protease inhibitors. J Acquir Immune Defic Syndr 26 Suppl 1: S34-50.

37. Mo H, King MS, King K, Molla A, Brun S, et al. (2005) Selection of resistance in protease inhibitor-experienced, human immunodeficiency virus type 1-infected subjects failing lopinavir- and ritonavir-based therapy: mutation patterns and baseline correlates. J Virol 79: 3329-3338. 
38. Colonno RJ, Thiry A, Limoli K, Parkin N (2003) Activities of atazanavir (BMS232632) against a large panel of human immunodeficiency virus type 1 clinical isolates resistant to one or more approved protease inhibitors. Antimicrob Agents Chemother 47: 1324-1333.

39. Kempf DJ, Isaacson JD, King MS, Brun SC, Xu Y, et al. (2001) Identification of genotypic changes in human immunodeficiency virus protease that correlate with reduced susceptibility to the protease inhibitor lopinavir among viral isolates from protease inhibitor-experienced patients. J Virol 75: 7462-7469.

40. Svedhem V, Lindkvist A, Bergroth T, Knut L, Sönnerborg A (2005) Diverse pattern of protease inhibitor resistance mutations in HIV-1 infected patients failing nelfinavir. J Med Virol 76: 447-451.

41. Chapman TM, Plosker GL, Perry CM (2004) Fosamprenavir: a review of its use in the management of antiretroviral therapy-naive patients with HIV infection. Drugs 64: 2101-2124.

42. Rodriguez-French A, Boghossian J, Gray GE, Nadler JP, Quinones AR, et al. (2004) The NEAT study: a 48-week open-label study to compare the antiviral efficacy and safety of GW433908 versus nelfinavir in antiretroviral therapynaive HIV-1-infected patients. J Acquir Immune Defic Syndr 35: 22-32.

43. Marcelin AG, Cohen-Codar I, King MS, Colson P, Guillevic E, et al. (2005) Virological and pharmacological parameters predicting the response to lopinavir-ritonavir in heavily protease inhibitor-experienced patients. Antimicrob Agents Chemother 49: 1720-1726.

44. Parkin NT, Chappey C, Petropoulos CJ (2003) Improving lopinavir genotype algorithm through phenotype correlations: novel mutation patterns and amprenavir cross-resistance. AIDS 17: 955-961.

45. Kagan RM, Shenderovich MD, Ramnarayan K, Heseltine PNR, et al. (2003) Emergence of a novel lopinavir resistance mutation at codon 47 correlates with ARV utilization. Antivir Ther. 8: S54

46. Parkin NT, Petropoulos CJ, Chappey C (2004) Isolated lopinavir resistance after virological rebound of a lopinavir/ritonavir-based regimen. Antivir Ther. 9: S79.

47. Busti AJ, Hall II RG, Margolis DM (2004) Atazanavir for the treatment of human immunodeficiency virus infection. Pharmacotherapy 24: 1732-1747.

48. Colonno R, Rose R, McLaren C, Thiry A, Parkin N, et al. (2004) Identification of $150 \mathrm{~L}$ as the signature atazanavir (ATV)-resistance mutation in treatment-naive HIV-1-infected patients receiving ATV-containing regimens. J Infect Dis 189: 1802-1810.

49. Colonno R, Parkin N, McLaren C (2004) Pathways to atazanavir resistance in treatment-experienced patients and impact of residue 50 substitutions [abstract 656]. Program and Abstracts of the11th Conference on Retroviruses and Opportunistic Infections. San Francisco, CA.

50. Coakley E, Mass M, Parkin N (2005) Atazanavir Resistance in a Protease Inhibitor-naïve Patient Treated with Atazanavir/Ritonavir Associated with Development of High-level Atazanavir Resistance and the N88S Mutation in Protease. Program and Abstracts of the12th Conference on Retroviruses and Opportunistic Infections. Boston, MA.

51. Croom KF, Keam SJ (2005) Tipranavir: a ritonavir-boosted protease inhibitor. Drugs 65: 1669-1677.

52. Baxter JD, Schapiro JM, Boucher CA, Kohlbrenner VM, Hall DB, et al. (2006) Genotypic changes in human immunodeficiency virus type 1 protease associated with reduced susceptibility and virologic response to the protease inhibitor tipranavir. J Virol 80: 10794-10801.

53. Hicks CB, Cahn P, Cooper DA, Walmsley SL, Katlama C, et al. (2006) Durable efficacy of tipranavir-ritonavir in combination with an optimised background regimen of antiretroviral drugs for treatment-experienced HIV-1-infected patients at 48 weeks in the Randomized Evaluation of Strategic Intervention in multi-drug reSistant patients with Tipranavir (RESIST) studies: an analysis of combined data from two randomised open-label trials. Lancet 368: 466-475.

54. Schapiro JM, Scherer J, Boucher CA, Baxter JD, Tilke C, et al. (2010) Improving the prediction of virological response to tipranavir: the development and validation of a tipranavir-weighted mutation score. Antivir Ther 15: 1011-1019.

55. Baxter JD, Kumar P, Ruane P (2010) Enhanced susceptibility of human immunodeficiency virus type- 1 to tipranavir in treatment-experienced patients. Infectious Diseases in Clinical Practice 18: 359-366.

56. De Meyer S, Azijn H, Surleraux D, Jochmann D, Tahri A, et al. (2005) TMC114, a novel human immunodeficiency virus type 1 protease inhibitor active against protease inhibitor-resistant viruses, including a broad range of clinical isolates. Antimicrob Agents Chemother 49: 2314-2321.

57. De Meyer S, Vangeneugden T, Lefebvre E, Azijn H, I De Baere, et al. (2006) Phenotypic and genotypic determinants of resistance to TMC114: pooled analysis of POWER 1, 2, and 3. Antivir Ther. 11: S83.

58. De Meyer S, Descamps D, Van Baelen B, Lathouwers E, Cheret A, et al. (2009) Confirmation of the negative impact of protease mutations $147 \mathrm{~V}, 154 \mathrm{M}, \mathrm{T} 74 \mathrm{P}$ and $184 \mathrm{~V}$ and the positive impact of protease mutation V82A on the virologic response to darunavir/ritonavir. Antivir Ther. 14: A147.

59. Descamps D, Lambert-Niclot S, Marcelin AG, Peytavin G, Roquebert B, et al. (2009) Mutations associated with virologic response to darunavir/ritonavir in HIV-1-infected protease inhibitor-experienced patients. J Antimicrob Chemother. 63: 585-592.

60. De Luca A, Philippe F, Dunn D, Zazzi M, Wensing A, et al. (2016) Improved darunavir genotypic mutation score predicting treatment response for patients infected with HIV-1 subtype $B$ and non-subtype $B$ receiving a salvage regimen. J Antimicrob Chemother 71: 1352-1360.

61. Winters MA, Baxter JD, Mayers DL, Wentworth DN, Hoover ML, et al. (2000) Frequency of antiretroviral drug resistance mutations in HIV-1 strains from patients failing triple drug regimens. Antivir Ther 5: 57-63.

62. Hertogs K, Bloor S, Kemp SD, Van den Eynde C, et al. (2000) Phenotypic and genotypic analysis of clinical HIV-1 isolates reveals extensive protease inhibitor cross-resistance: a survey of over 6000 samples. AIDS 14: 1203-1210.

63. Ziermann R, Limoli K, Das K, Arnold E, Petropoulos CJ, et al. (2000) A mutation in human immunodeficiency virus type 1 protease, N88S, that causes in vitro hypersensitivity to amprenavir. J Virol $74: 4414-4419$.

64. Schooley R, Haubrich R, Thompson M (2003) Effect of Amprenavir hypersusceptibility on the response to APV/ritonavir-based therapy in ARTexperienced adults selected by baseline susceptibility (ESS40006): 24-week data. Program and Abstracts of the10th Conference on Retroviruses and Opportunistic Infections. Boston, MA.

65. Weinheimer S, Discotto L, Friborg J, Yang H, Colonno R (2005) Atazanavir signature $150 \mathrm{~L}$ resistance substitution accounts for unique phenotype of increased susceptibility to other protease inhibitors in a variety of human immunodeficiency virus type 1 genetic backbones. Antimicrob Agents Chemother. 49: 3816-3824.

66. Baxter JD, Bhatti L, Coakley E, Bartczak J, McDonough M, Vinisko R, Pilliero PJ, et al. (2010) Susceptibility of HIV-1 to tipranavir and other antiretroviral agents in treatment-experienced patients: The UTILIZE Study. Current HIV Research. 8: 347-354.

67. Kuritzkes DR (2004) Preventing and managing antiretroviral drug resistance AIDS Patient Care STDS 18: 259-273.

68. Rodes B, García F, Gutierrez C, Martinez-Picado J, Aguilera A, et al. (2005) Impact of drug resistance genotypes on CD4+ counts and plasma viremia in heavily antiretroviral-experienced HIV-infected patients. J Med Virol 77: 23-28.

69. Lawrence J, Hullsiek KH, Thackeray LM, Abrams DI, Crane LR, et al. (2006) Disadvantages of structured treatment interruption persist in patients with multidrug-resistant HIV-1: final results of the CPCRA 064 study. J Acquir Immune Defic Syndr 43: 169-178.

70. Liu TF, Shafer RW (2006) Web resources for HIV type 1 genotypic-resistance test interpretation. Clin Infect Dis 42: 1608-1618.

71. Shafer RW, Rhee SY, Pillay D, Miller V, Sandstrom P, et al. (2007) HIV-1 protease and reverse transcriptase mutations for drug resistance surveillance. AIDS 21: 215-223 